\title{
Rheumatoid arthritis: too high a price for corticosteroids
}

\author{
Anca-Maria Dima', Madalina Duna', Lavinia Stanescu', Traian-Costin Mitulescu², \\ Denisa Predeteanu ${ }^{1}$ \\ ${ }^{1}$ Department of Internal Medicine and Rheumatology, "Sf. Maria" Clinical Hospital, Bucharest, Romania \\ 2Department of Ophtalmology, Bucharest Emergency University Hospital, \\ "Carol Davila" University of Medicine and Pharmacy, Bucharest, Romania
}

\begin{abstract}
Rheumatoid arthritis (RA) is a chronic rheumatic systemic disease characterized by proliferative synovitis, with cartilage and bone destruction, affecting joint functions with serious effects on the patient's quality of life. Heterogenicity of the disease, complications and comorbidities are important in choosing the treatment.

Corticosteroids as treatment of RA represent an important issue both through their positive effects on inflammation and its consequences and through their negative effects especially when used excessively. Complications due to excessive corticosteroid therapy such are infections, osteoporotic bone fractures and cataract represent a very high burden as our title suggests. Hence, the need for this presentation of a clinical case in order to illustrate the side effects of such a therapy as well as the choice of the best solution.

The evolution of our patient's disease demonstrates that our approach is good and our results encourage us to continue in this way.
\end{abstract}

Keywords: corticosteroids, rheumatoid arthritis, side effects

\section{INTRODUCTION}

Rheumatoid arthritis (RA) is the most common systemic inflammatory rheumatic disease characterized by deforming, destructive and debilitating arthropathy. RA incidence and prevalence are higher in women than in men; the disease affects about $1 \%$ of the general population with an incidence of $0.5 / 1000$ in women and of $0.2 / 1000$ in men and a prevalence of about $1.7 \%$ in women and of $0.7 \%$ in men. Compared to the general population RA patients have a higher risk of death, particularly from infection and gastrointestinal disease (1). Three important concepts have become standard of care in treating RA: development of new drugs such are biologic agents, therapeutic strategies represented by combination of different drugs and treat to target (T2T) that means to treat the early disease in order to obtain remission and established disease in order to obtain low-disease activity (2,3). Many conventional synthetic (cs) and biologic (b) disease-modifying antirheumatic drugs (DMARDs) (4) are used in the treatment of RA, and the best strategy using csD-
MARDs and bDMARDs in combination with shortterm corticosteroids is applied to treat patients according to T2T. Corticosteroid therapy has an anti-inflammatory effect, thus decreasing the signs and symptoms of the disease, but its excessive use may cause considerable side effects (5). We have exemplified all this by presenting a case of a patient with severe RA, excessively treated with corticosteroids, which resulted in several life-threatening complications. The correct therapeutic approach, as indicated by the latest recommendations regarding the maximal dosage of DMARDs, and the progressive decrease of corticosteroids had a favorable effect. Collaboration between specialists has proved fruitful for the correct management of the disease, contributing to a better quality of life of the patient.

\section{CASE REPORT}

We present the case of a female patient, aged 59, smoker for over 20 years, professional driver, diagnosed with seronegative RA approximately 17 years ago and recently admitted to our department for 
evaluation of the disease and optimization of the treatment. The patient has complained of inflammation of bilateral wrists and metacarpo-phalangeal (MCP) and proximal interphalangeal (PIP) joints of the hands accompanied by morning stiffness for more than 1 hour.

The history of the patient is not relevant, except an early menopause at the age of 42 , without any hormonal treatment.

The rheumatic disease of the patient began in 2000, with pain and swelling in the right knee; X-ray of the right knee aroused doctor's suspicion of a torn meniscus and consequently arthroscopy was performed. Three months later, pain and swelling reappeared in both knees and a total knee synovectomy allowed the analysis of the synovial membrane. As the inflammation of the synovial membrane was considered nonspecific, the patient was referred to the rheumatologist who indicated dexamethasone i.v. followed by nonsteroidal anti-inflammatory drugs (NSAIDs) which caused improvement in about 3 weeks.

Subsequent pain, swelling and deformities of the hands appeared in 2004, when the diagnosis of RA was made and methotrexate (MTX) therapy up to 15 $\mathrm{mg} /$ week was initiated. As remission was not achieved with that medication, biologic treatment with infliximab (IFX) $5 \mathrm{mg} / \mathrm{kg}$ every 8 weeks was added in 2005, initially to MTX and subsequently to leflunomide (LFN), and therapeutic response was good.

In autumn 2008 the disease was in remission, so the attending physician decided spacing biological therapy but that caused a decrease in the overall therapeutic response. At the end of 2008 the patient suffered a right femur fracture after a fall from her own height and a osteosynthesis with gamma nail was performed to immobilize the fractured hip. At that moment, the rheumatologist considered the disease unresponsive to treatment and decided to switch from IFX to Etanercept (ETN) $50 \mathrm{mg} /$ week, which was stopped after 7 months, due to repeated allergic reactions at the injection site. In 2009 there was a switch from ETN to Rituximab (RTX) 1,000 mg x2/ cycle, because disease activity was maintained at the same high level. After the first perfusion of the second cycle the patient suffered a second femur fracture at the level of the left hip, requiring the same surgical treatment as the first fracture. Difficulty in walking prevented her from going to hospital to continue treatment with RTX.
From 2009 to 2013 the patient continued the treatment with LFN, and, as she felt better under treatment with corticosteroids, she administered on her own decision dexamethasone i.m. initially every 3-4 days and then daily. Contrary to the doctors' warnings about self-medication, especially with corticosteroids, the patient took this decision which brought about severe complications.

Therefore in 2013 the patient presented to our clinic for the first time carried on a stretcher, complaining of severe low back pain and presenting a painful ulceration of the right leg that had evolved for 5 months, nonresponsive to local treatment with a disinfectant. The clinical examination of peripheral arteries was normal and additional investigations such as Doppler arterial ultrasound or arteriography were not possible at that moment. The lab tests performed at that time showed an important inflammatory syndrome with leukocytosis $(>20,000 / \mathrm{mmc})$, high level of ESR $(109 \mathrm{~mm} / \mathrm{h})$ and high level of C reactive protein $(320 \mathrm{mg} / \mathrm{dl})$ together with altered renal function with high level of urea $(134 \mathrm{mg} / \mathrm{dl})$, uric acid $(20,50 \mathrm{mg} / \mathrm{dl})$ and creatinine $(4.80 \mathrm{mg} / \mathrm{dl})$ probably through extrarenal mechanisms. The patient did not have a histopathological examination and exclusion of pyoderma gangraenosum, which is a nonfectious neutrophilic dermatosis, was not possible as that moment. The lumbar resonance magnetic imaging (MRI) raised the suspicion of bacterial osteomyelitis of the lumbar spine of unknown etiology. In order to rule out sepsis, procalcitonin was indicated and the result was negative, all the more the patient had not fever. After 2 weeks of antibiotic treatment according to the result of antibiogram of the leg ulceration, the patient was referred to the Department of Neurosurgery of the "Bagdasar-Arseni" Hospital for a vertebral biopsy. There, the ulceration of the right leg was demonstrated to be caused by Pseudomonas aeruginosa and after a long treatment with antibiotics, chosen accordingly to bacteriological data, a skin graft was performed and the result was favorable. As antibiotic treatment had a good effect also on her lumbar pain, vertebral biopsy was no longer considered and surgery was performed consisting of metal plate fastened with screws at the level of L2-L5, with subsequent improvement of posture and gait. As the lumbar MRI showed osteoporotic lumbar spine, multiple osteoporotic vertebral fractures and lumbar scoliosis, osteodensitometry was ordered and revealed severe osteoporosis (lumbar spine L1-L4: T score -4.6, BMD:0.599 $\left.\mathrm{g} / \mathrm{cm}^{2}\right)$, 
probably with an important contribution of excessive corticosteroids.

Back to our clinic in 2014 for the reevaluation of treatment, the disease being intensely active (DAS $28=6.9$ ) with significant impairment of life quality $(\mathrm{HAQ}=2)$, maximal doses of MTX (20 mg/week) with small doses of corticosteroids with prednisone (Pred) $(10 \mathrm{mg} /$ day $)$ were indicated. Due to severe rheumatoid involvement of the left knee through secondary osteoarthritis and the deformity of the knee affecting posture and walking, left knee replacement with metallic prosthesis was performed with good results. The subsequent evaluation showed low disease activity (DAS $28=2.8$ ) with moderate influence on life quality $(\mathrm{HAQ}=1)$ and therefore the decision of maintaining the dose of MTX $(20 \mathrm{mg} /$ week) and decreasing the dose of Pred ( $5 \mathrm{mg}$ /day) was indicated. At the same time, bisphosphonates (risedronate) in association with calcium and vitamin D were also recommended for the treatment of severe osteoporosis.

At the same time, the patient presented eye problems and an ophthalmologic exam performed at the Bucharest Emergency University Hospital made the diagnosis of bilateral corticosteroid cataract; lens surgery was successfully performed.

Between 2014 and 2017 the patient was lost to follow up; she came back to our clinic in May 2017 saying that she discontinued the treatment with MTX

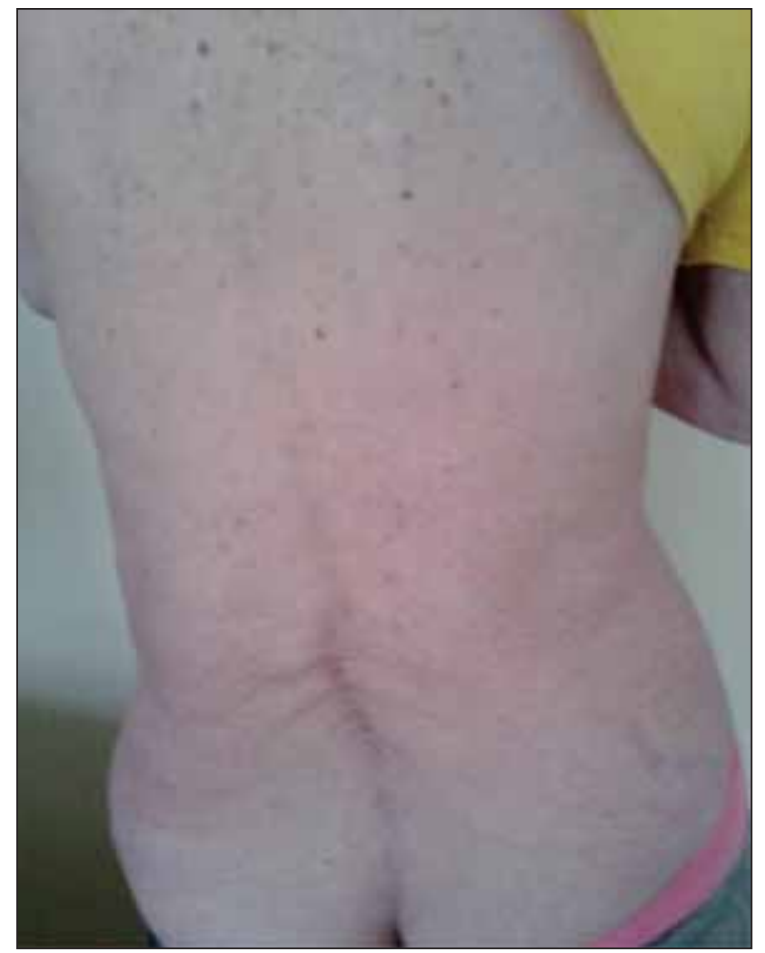

FIGURE 1. Lumbar post-surgery scar but she had maintained the treatment with Pred $(10 \mathrm{mg} /$ day) for RA and the treatment with bisphosphonate for osteoporosis.

The clinical examination showed a patient with a good general state of health with relatively good posture and lumbar post-surgery scar (Fig. 1) with a noticeable inferior limbs inequality with moderate swellings of the wrists (left > right) and MCP joints, together with important interosseous muscle atrophy (Fig. 2) and chronic ulcerative lesions in both legs (right $>$ left) (Fig. 3).

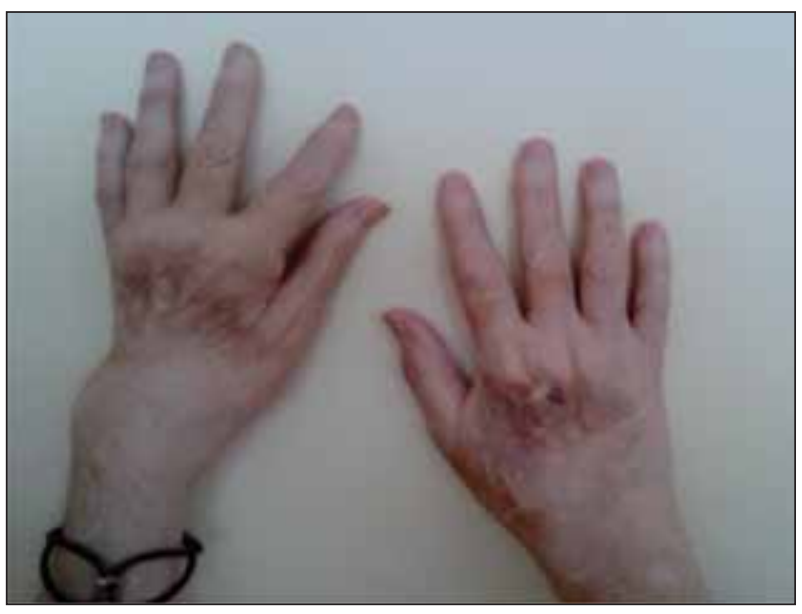

FIGURE 2. Clinical aspect of the hands

Laboratory tests performed between 2013 and 2017 revealed normal blood counts and confirmed seronegativity of the disease, even if at the beginning of the disease the level of rheumatoid factor (RF) was slightly increased (23.7 UI/L versus 9.4 $\mathrm{UI} / \mathrm{L})$; the tests for anti-citrullinated protein antibodies (ACPA) were invariably negative. The lab tests showed a favorable evolution of the nonspecific inflammatory syndrome with the normalization of renal function (Table 1).

TABLE 1. Laboratory results of the patient (2013-2017)

\begin{tabular}{|l|c|c|c|}
\hline & February 2013 & April 2014 & May 2017 \\
\hline Hemoglobin (g/dl) & $\mathbf{1 1 , 1}$ & 12,1 & 11,6 \\
\hline Thrombocytes $\left(\mathrm{mm}^{3}\right)$ & $\mathbf{4 2 0 , 0 0 0}$ & 220,000 & 170,000 \\
\hline WBC $\left(\mathrm{mm}^{3}\right)$ & $\mathbf{2 0 , 3 0 0}$ & 8,810 & $\mathbf{7 , 8 0 0}$ \\
\hline ESR $(\mathrm{mm} / \mathrm{h})$ & $\mathbf{4 8}$ & 23 & $\mathbf{5 2}$ \\
\hline CRP $(\mathrm{mg} / \mathrm{l})$ & $\mathbf{1 8 4 , 3}$ & $\mathbf{4 1 , 5}$ & $\mathbf{1 1 4}$ \\
\hline Fibrinogen $(\mathrm{mg} / \mathrm{dl})$ & $\mathbf{4 9 6 , 0}$ & 357,0 & $\mathbf{4 5 7 , 0}$ \\
\hline RF $(\mathrm{Ul} / \mathrm{l})$ & $\mathbf{2 3 , 7}$ & 9,4 & $<20$ \\
\hline ACPA (U/ml) & $<0,5$ & 11,79 & 1,86 \\
\hline Glucose $(\mathrm{mg} / \mathrm{dl})$ & $\mathbf{1 2 3}$ & 64 & 87 \\
\hline Creatinine $(\mathrm{mg} / \mathrm{dl})$ & $\mathbf{1 , 1 9}$ & 0,97 & $\mathbf{1 , 2}$ \\
\hline Urea $(\mathrm{mg} / \mathrm{dl})$ & $\mathbf{1 3 4}$ & 0,97 & $\mathbf{4 7}$ \\
\hline Uric acid $(\mathrm{mg} / \mathrm{dl})$ & $\mathbf{2 0 , 5}$ & - & $\mathbf{8 , 4 8}$ \\
\hline GOT $(\mathrm{U} / \mathrm{l})$ & 30 & 11 & 10 \\
\hline GPT $(\mathrm{U} / \mathrm{l})$ & 47 & 5 & 6 \\
\hline
\end{tabular}



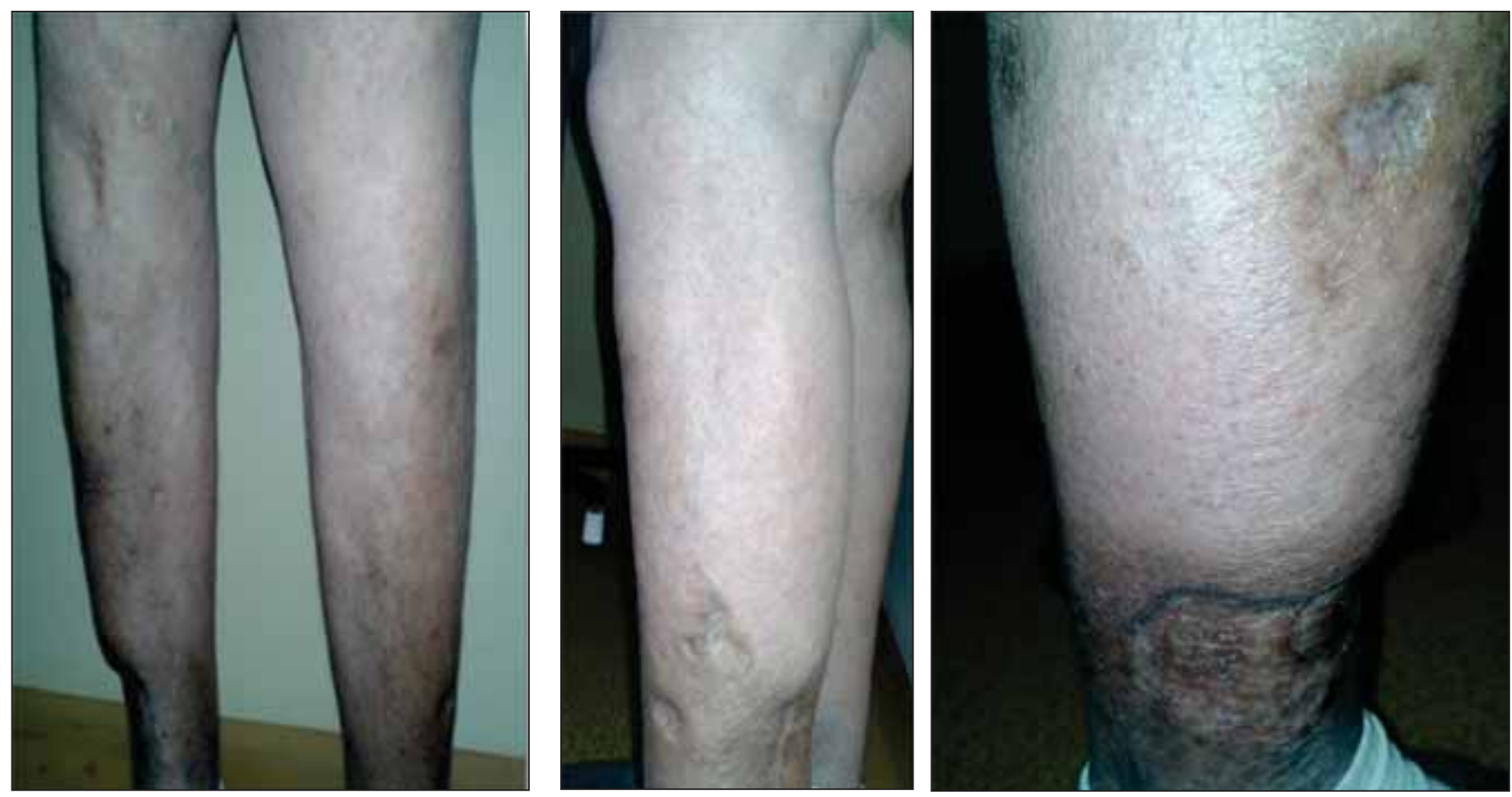

FIGURE 3. Chronic ulcerative lesions in both legs (right > left)

X-ray of the hands showed periarticular osteopenia, narrowing of the joint spaces and the presence of multiple erosions (Fig. 4) and X-ray of the pelvis presented the bilateral metallic plate with gamma nail inserted for bilateral hip fractures (Fig. 5). X-ray of the knees showed metallic prosthesis of the left knee (Fig. 6) inserted for secondary osteoarthritis of the left knee with involvement of the posture and gait.

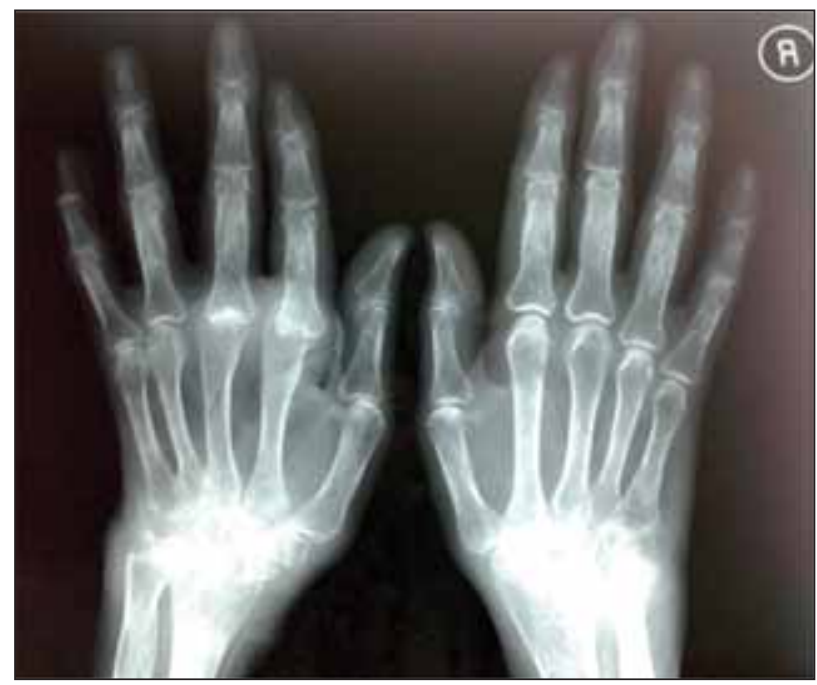

FIGURE 4. X-ray of the hands: periarticular osteoporosis, joint narrowing, erosions

Lumbar spine magnetic resonance imaging (MRI) performed in 2013 before spine surgery showed scoliosis, vertebral fractures and L4-L5, L5S1 spondylodiscitis (Fig. 7); in 2015, MRI per-

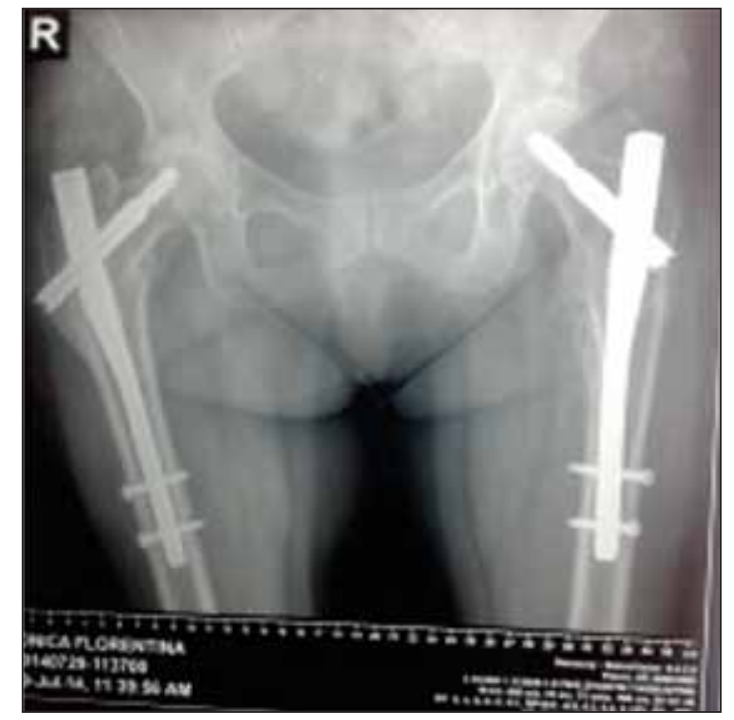

FIGURE 5. X-ray of the pelvis: bilateral metallic plate with gamma nail

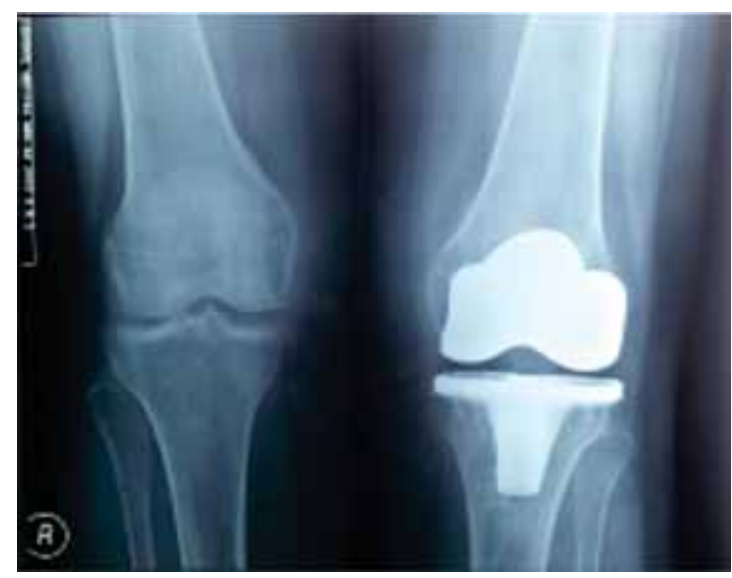

FIGURE 6. X-ray of the knees: metallic prosthesis of the left knee 

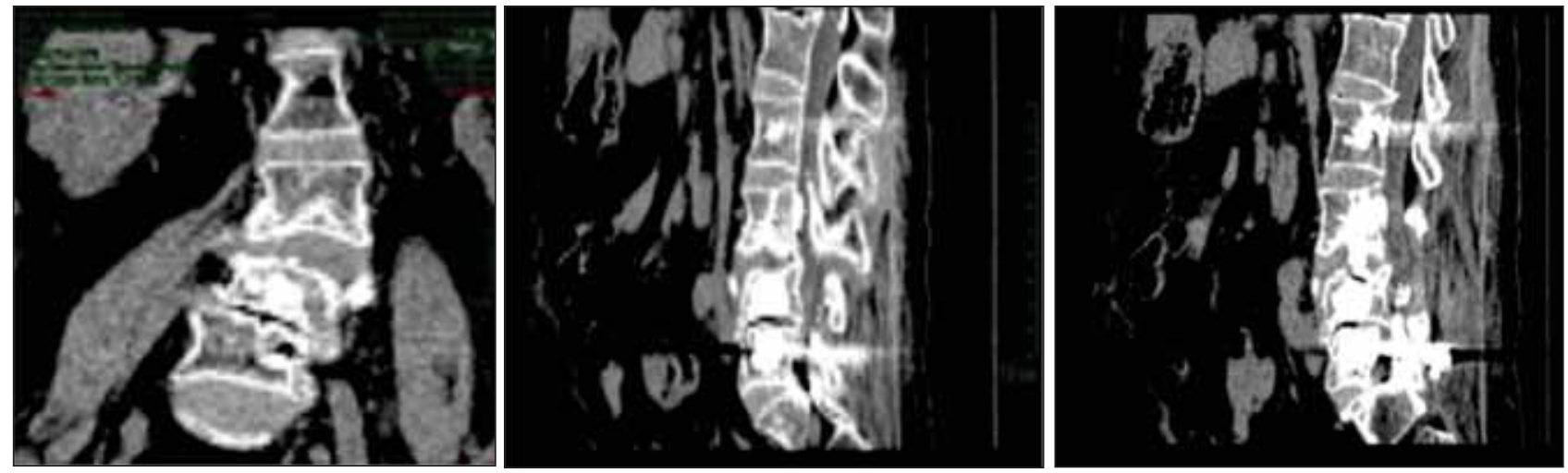

FIGURE 7. Lumbar MRI before spine surgery (2013): scoliosis, vertebral fracture, spondylodiscitis

formed after spine surgery only showed metallic plate with 2 screws, the inferior screw being fractured (Fig. 8).

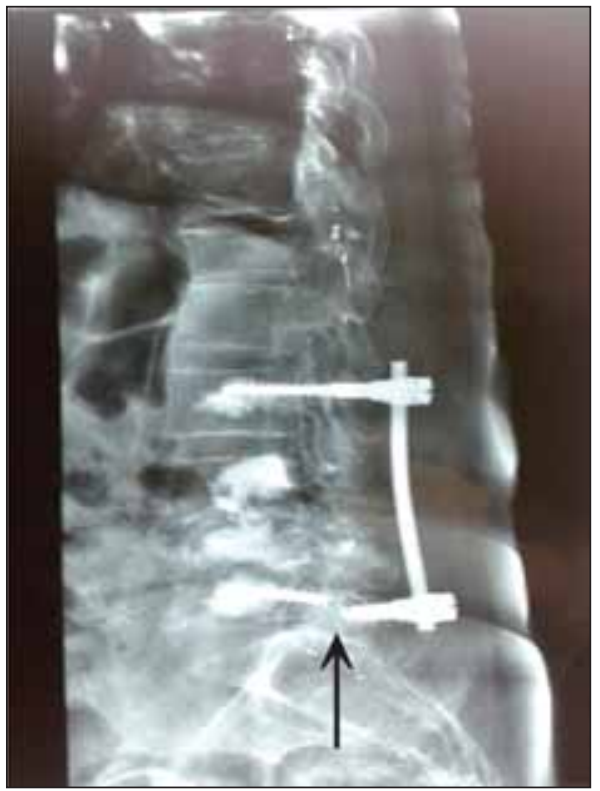

FIGURE 8. Lumbar MRI after spine surgery (2015): metalic plate with 2 screws, fractured inferior screw

Considering all these clinical and paraclinical data, the positive diagnosis of the patient was:

1. Seronegative RA, stage III (Steinbrocker functional classification) nonresponsive or intolerant to many csDMARDs (MTX, LFN) and many bDMARDS (IFX, ETN, RTX) at present under treatment with MTX $(20 \mathrm{mg} /$ week) and Pred (10 mg/day) with good response.

2. Severe osteoporosis with multiple pathogenic mechanisms (early menopause, immobilization, smoking) worsened by excessive selfadministration of corticosteroids, complicated with multiple fractures which needed surgery and treatment with bisphosphonates (risedronate), calcium and vitamin D.
3. Infection spondylodiscitis treated by implanting a plate with 2 screws, the lower one being subsequent fractured.

4. Multiple ulcerations of the legs due to infection with Pseudomonas aeruginosa with favorable evolution under antibiotics.

5. Total metallic prosthesis of the left knee for secondary osteoarthritis.

6. Bilateral operated glucocorticoid-induced cataract.

7. Reversible renal failure through extrarenal mechanisms and residual hyperuricemia, with indication of treatment with allopurinol.

We have decided to strictly monitor the patient; if the disease will not respond to treatment with MTX and low doses of corticosteroids, we shall add a new bDMARD.

\section{DISCUSSION}

We have presented a case report of a patient with severe long-standing seronegative RA, nonresponsive or intolerant to many csDMARDS and bDMARDs, excessively treated with corticosteroids, resulting in various life-threating complications, many of them requiring surgery.

Corticosteroids represent a class of drugs much used in RA, especially for the effects on inflammation and in reducing the signs and symptoms of the disease. The symptomatic effect of corticosteroids appears soon, continues during treatment and does not influence the pathogenic process of the disease (6).

According to literature and our own clinical practice, there is now a change in the old concept of using high doses of corticosteroids in RA, due to multiple side effects; the most recent recommendations suggest low or moderate doses of corticosteroids, avoiding serious side effects. Low-dose corti- 
costeroid therapy (Pred 5-10 mg/day) for a limited period, in combination with standard therapy is considered basic strategy in early RA (in the first 2 years of treatment) (7). The combination therapy of a low dose of corticosteroids with cs or bDMARDs represents the gold standard in most therapeutic protocols involving treatment, according to the concept of T2T, both in early and long-standing RA. Medium and high doses of corticosteroids are used as „,bridge therapy“ when starting DMARDs, until these become effective. Intra-articular steroid injections also can be effective in reducing local inflammation (8).

Side effects of corticosteroids are more likely to occur when these are used as regular treatment over a longer period of time. This is why physicians try to limit the long-term reliance on corticosteroids for RA treatment. Following a strategy in Ovid Medline search (January 2000 - December 2012) using words like Population (PR), Intervention (GC), comparator (comparison), Outcome (AR) were identified in 1031 papers, 26 observational studies and 6 systematic reviews of adverse effects of GC. The major side effects consist of bone loss, increased risk of cardiovascular events and infection, fatigue, increased blood sugar, cataracts, mood disruption including depression, anxiety and insomnia. However the risk appears to be dose-dependent (9).

Osteoporosis is the most serious adverse effects experienced by patients receiving long term corticosteroid therapy. Bone loss occurs soon after the initiation of corticosteroid treatment (common during the first 6-12 months) and results from a complex mechanism involving osteoblastic suppression and increased bone resorption. Patients on corticosteroid therapy are at increased risk of sustaining fractures and fracture risk is dose-dependent (10). Risk factors for fractures in patients treated with $\mathrm{GC}$ are divided into individual factors (high daily doses/cumulative dose increased, age extremes $<15$ year $>60$ years, postmenopausal status, low pre-existing bone mineral density, pre-existing fractures from minimal trauma, type of inflammatory disease, prolonged immobilization) and general factors (female, white race, low bone mineral index, diet with low intake of calcium and vitamin D, smoking, family history of osteoporosis) (11).

A study of a cohort of patients in RA Danish Registry (DREAM) followed predictors of risk of infection treated with anti-TNF. Thus, after 5 years of follow-up, 128 of 2044 (6.3\%) patients developed a serious infection first with a total of 141 serious in- fections. The incidence rate in the first year after start of TNF inhibiting therapy was 4.57 first serious infections per 100 patient-years and 2.91 per 100 patient-years over 5 years. Age, corticosteroids use, visual analogue scale (VAS) pain, HAQ, tender joint count 28 joints (TJC28) and the presence of comorbidities were significant predictors for developing a serious infection during TNF inhibiting therapy in the multivariate model (12).

Recently, European League Against Rheumatism (EULAR) recommendations for the managing of RA with synthetic and biological disease-modifying antirheumatic drugs - 2016 update, established that short term glucocorticoids should be consider when initiating or changing csDMARDs in different dose regimens and routes of administrations, but should be tapered as rapidly as clinically feasible (13).

The presentation of this case is very important for clinical practice, because it shows both the multiple corticosteroid-induced complications, due to high doses of corticosteroids used as self-medication (skin ulcerations with superimposed infections, severe multiple osteoporosis fractures surgically solved with metal implants and bilateral cataract), and the need for adapting the therapeutic strategy, strictly monitoring disease activity and treatment safety.

\section{CONCLUSIONS}

1. Corticosteroids represent an important and effective treatment for RA when administered in correct recommended doses.

2. Rheumatologists should weigh the pros and cons of corticosteroid treatment in the therapeutic strategy of RA.

3. Excessive doses of corticosteroids for a long period bring about complications which may be even life threatening.

4. Patients should be warned that self-administration of corticosteroids, as well as any changes without the doctor's advice, may be dangerous with dramatic results.

5. The correct doses of corticosteroids within the therapeutic strategy of RA and close monitoring of patient proved to give good results.

6. Management of complex cases such as the one presented requires an interdisciplinnary approach to solve specific problems by various specialists. 
7. The general practitioner has the obligation to follow up the patient with RA treated with DMARDs and low doses of corticosteroids in order to diminish the side effects of an excessive and uncontrolled treatment.

Conflict of interest: none declared Financial support: none declared

\section{REFERENCES}

1. Kuo C-F., Luo S-F., See L-C. et al. Rheumatoid arthritis prevalence, incidence and mortality rates: a nationwide population study in Taiwan. Rheum Int 2013; 33(2):355-60 .

2. Bijlsma J.W. Optimal treatment of rheumatoid arthritis: EULAR recommendations for clinical practice. Pol Archy Med Wewn 2010 Sep; 120(9):347-53.

3. Smolen J.S., Aletaha D., Bijlsma J.W. et al for the T2T Expert Committee. Treating rheumatoid arthritis to target: recommendations of an international task force. Ann Rheum Dis 2010;69:631-37

4. Smolen J.S., van der Heijde D., Machold K.P. et al. Proposal for a new nomenclature of disease-modifying antirheumatic drugs. Ann Rheum Dis 2014Jan 26;73(1):3-5 .

5. Townsend H.B., Saag K.G. Glucocorticoid use in rheumatoid arthritis: benefists, mechanisms and risks. Clin Exp Rheumatol 2004;22(Suppl 35):S77-S82.

6. Bălănescu Andra. Poliartrita reumatoidă în „Esențialul în reumatologie“ Ruxandra lonescu (coord), Ediția I, Ed. Amaltea, Bucureşti, 2006, 214-251.

7. Strand V., Simon L.S. Low dose glucocorticoids in early rheumatoid arthritis. Clin Exp Rheumatol 200321 (5 Suppl31): S186-90.
8. Hoes J.N., Jacobs J.W., Buttgereit F., Bijlsma J.W. Current view of glucocorticoid co-therapy with DMARDs in rheumatoid arthritis Nat Rev Rheumatol 20106 (12): 693-702.

9. Ethgen O., de Lemos Esteves F., Bruvere O., Reginster J.Y. What do we know about the safety of corticosteroids in rheumatoid arthritis?. Curr Med Res Opin 2013, 29 (9): 1147-60.

10. Ionescu Ruxandra. Glucocorticoizii în „Esențialul în reumatologie“, Ruxandra lonescu (coord). Ediția a Il-a revizuită. Ed. Amaltea, Bucureşti, 2007. IV: 151-161.

11. Canalis E., Mazziotti G., Giustina A. et al. Glucocorticoid-induced osteoporosis: pathophysiology and therapy. Osteoporosis Int 2007; 18: 1319-22.

12. Van Dartel S.A., Fransen J., Kievit W. et al. Predictors for the 5-year risk of serious infections in patients with rheumatoid arthritis treated with anti-TNF therapy: a cohort study in the Duch Rheumatoid Arthritis Monitoring (DREAM) registry, Rheumatology (Oxford) 2013, 52 (6): 1052-7.

13. Smolen J.S., Landewe R., Bijlsma J. et al. EULAR recommendations for the management of rheumatoid arthritis with synthetic and biological disease-modifying antirheumatic drugs: 2016 update, Ann Rheum Dis, March 2017, http://ard.bmj.com/ content/early/2017/03/17/annrheumdis-2016-210715. 